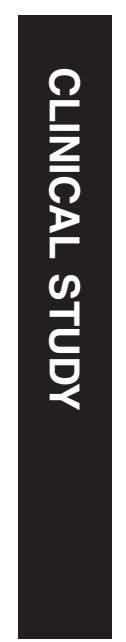

\section{Associations of aniseikonia with metamorphopsia and retinal displacements after epiretinal membrane surgery}

Y Ichikawa ${ }^{1,2}$, Y Imamura ${ }^{1}$ and $M$ Ishida ${ }^{1}$

\section{Introduction}

\section{${ }^{1}$ Department of Ophthalmology, Teikyo University School of Medicine, University Hospital Mizonokuchi, Kanagawa, Japan \\ ${ }^{2}$ Department of Ophthalmology, Saitama Medical University, Iruma, Japan}

Correspondence: Y Imamura, Department of Ophthalmology, Teikyo University School of Medicine, University Hospital Mizonokuchi, 3-8-3 Mizonokuchi Takatsuku, Kawasaki, Kanagawa 213-8507, Japan

Tel: +81 448443333 ;

Fax: +8144844 3201.

E-mail: yimamura.ny@ gmail.com

Received: 23 February 2017 Accepted in revised form: 5 July 2017

Published online:

22 September 2017

\begin{abstract}
Purpose To determine the correlation of the degree of aniseikonia with the retinal displacements and metamorphopsia in patients that have undergone successful epiretinal membrane (ERM) surgery. Methods Subjects were 28 eyes with an ERM in 28 patients. The New Aniseikonia Test (NAT) and M-CHARTS were used to quantify the degree of preoperative and postoperative aniseikonia and metamorphopsia. We also evaluated the distance between the intersections of 2 sets of retinal vessels situated vertically or horizontally by using spectral-domain optical coherence tomography (SD-OCT) images in 28 patients.

Results The vertical score of M-CHARTS (MV) was not significantly improved, but the horizontal score of M-CHARTS (MH) was significantly improved at 1 week, 1 month, and 3 months postoperatively. The preoperative NAT score was significantly correlated with the preoperative MH. The NAT score at 3 months was significantly correlated with the $\mathrm{MH}$ at 3 months and the MV at 3 months. The preoperative NAT score was significantly correlated with the ratio of the vertical retinal displacement at 1 month and at 3 months after surgery. However, the NAT scores did not improve significantly at any postoperative times.

Conclusions The degree of aniseikonia was significantly correlated with the degree of metamorphopsia and the tangential displacement of the retina after ERM surgery. Aniseikonia is difficult to improve and metamorphopsia may be a more sensitive parameter to detect the functional recovery after successful ERM surgery.

Eye (2018) 32, 400-405; doi:10.1038/eye.2017.201; published online 22 September 2017
\end{abstract}

Aniseikonia and metamorphopsia are major complaints of patients with an epiretinal membrane (ERM). ${ }^{1-4}$ Aniseikonia is an ocular condition in which there is a significant difference in the perceived size of images by the two eyes, while metamorphopsia is a type of visual defect in which the shapes of objects are distorted and straight lines appear wavy. Vitrectomy with ERM peeling is the standard treatment for an ERM, however even after successful surgery, many patients still complain about aniseikonia or metamorphopsia or both.

Earlier, we examined the displacement of the retina after vitrectomy for an ERM and found a significant correlation between the distance of tangential retinal displacement and the degree of metamorphopsia as quantified by M-CHARTS (Inami Co, Tokyo, Japan). ${ }^{5}$

We hypothesized that if the metamorphopsia was induced by tangential movements of the inner retina, then the aniseikonia associated with an ERM may also be due to the same mechanism. To test this hypothesis, we determined the degree of aniseikonia and metamorphopsia and the retinal displacements before and after ERM surgery. We then determined the correlations of the degree of aniseikonia with the retinal displacements and the degree of metamorphopsia.

Materials and methods

\section{Patient selection}

We retrospectively analyzed the findings of 28 consecutive patients who had undergone vitrectomy with ERM peeling and had had their aniseikonia and metamorphopsia measured before and after the surgery. ${ }^{5}$ The participants 
were the part of the ERM patients whose data on the metamorphopsia have been published. ${ }^{5}$ Patients with $<2.0 \mathrm{D}$ of anisometropia before and 3 months after surgery were included. The study was approved by the Institutional Review Board of Teikyo University School of Medicine, and they conformed to the tenets of the Declaration of Helsinki. An informed consent for the surgery and measurements was obtained from each of the patients.

\section{Vitrectomy surgical procedures}

Surgery was performed by 2 vitreoretinal surgeons from 1 February 2013 to 28 February 2014 at the Teikyo University School of Medicine, University Hospital in Mizonokuchi (Kanagawa, Japan). The surgical procedures were same as reported in detail. ${ }^{5}$ All patients underwent standard 3-port vitrectomy (23 G or $25 \mathrm{G}$ ). Internal limiting membranes (ILM) were peeled in all the cases.

\section{Evaluation of retinal displacement with SD-OCT}

The methods of selecting the intersections of two retinal vessels in the near-infrared (IR) images of the Spectralis (Heidelberg Engineering, Dossenheim, Germany) were the same as described. ${ }^{5}$ Briefly, the points of intersection were chosen so that a line which passed from the superior point to the inferior was approximately vertical, and a line, which passed from the nasal point to the temporal was approximately horizontal, keeping the line passing through the center of macula. The distance between each set of intersections was measured with the caliper function embedded in the Spectralis OCT instrument. Both the vertical and horizontal distances were measured preoperatively, and at 1 week, 1 month, and 3 months after the surgery. The ratio of the changes in the distances, that is, preoperative distance/postoperative distance (\%), was used for the statistical analyses.

\section{Quantification of aniseikonia}

The New Aniseikonia Test (NAT; Handaya, Tokyo, Japan) was used to quantify the degree of aniseikonia. This test measures the degree of aniseikonia by dissociating the two eyes with a spectacle with a green filter before the right eye and a red filter before the left eye. The patient examined a book containing pairs of red and green semicircles. The diameter of the left red semicircle was constant at $4 \mathrm{~cm}$, and the measurements were made at $40 \mathrm{~cm}$ distance so the fixed left semicircle subtended a visual angle of $5.7^{\circ}$. The red and green semicircles were arranged in pairs with the size of the right green semicircle varying from 1 to $24 \%$ in increments of $1 \%{ }^{6}$ The subject wearing the red-green spectacles viewed the plates with the affected eye viewing the right green semicircle in each pair, and for the unaffected eye to see the left red semicircle as a reference. The subject indicated the pair in which the two semicircles appear to be of equal size. The difference (in \%) in the sizes of the semicircles in that pair represented the percentage of aniseikonia. We defined an aniseikonia of $+2 \%$ or more as macropsia and of $-2 \%$ or less as micropsia. ${ }^{7,8}$

\section{Quantification of metamorphopsia}

The degree of metamorphopsia was quantified by using M-CHARTS (Inami Co, Tokyo, Japan) as reported in detail. ${ }^{5}$ Briefly, the M-CHARTS consisted of plates with a straight line (0 degree) or dotted lines with interval between the dots ranging from 0.2 to 2.0 degree of visual angle. If the patient reports that the fine dotted line is distorted, the dot interval is increased until the patient reports perceiving the dotted line as being straight. The M-CHARTS score was examined for both vertical and horizontal lines.

\section{Statistical analysis}

The best-corrected visual acuity (BCVA) measured with a Landolt $C$ chart was converted to the logarithm of minimum angle of resolution (logMAR) units for statistical analyses. Paired $t$ tests were used to determine the significance of differences in the visual acuity and retinal distances. The New Aniseikonia Test scores and M-CHARTS scores were compared with the Wilcoxon signed-ranks test. Spearman's correlation coefficient by rank test was used to determine correlations between the New Aniseikonia Test scores and the visual acuity, the M-CHARTS scores, and the retinal displacement. A $P$ value $<0.05$ was considered statistically significant.

\section{Results}

There were 28 eyes of 28 patients (17 women) who met the inclusion criteria. Their mean age was $68.3 \pm 7.1$ years (range, 49-84), and 20 (71\%) had macropsia and 8 (29\%) had no aniseikonia. The mean preoperative refractive error (spherical equivalent) was $-1.96 \pm 2.66 \mathrm{D}$, and 2 eyes had more than $6.0 \mathrm{D}$ of myopia. The mean duration of the symptoms before surgery was $14.8 \pm 16.5$ months (range, 2-60). Vitrectomy was performed with $23-\mathrm{G}$ instruments in 12 eyes and 25-gauge instruments in 16 eyes.

Simultaneous cataract surgery was performed on 24 eyes and 4 were pseudophakic before the surgery.

Triamcinolone acetonide was used to make the ERM and ILM more visible in 4 of the 28 eyes. A posterior hyaloid detachment was present in 26 eyes, and no serious 
complications occurred during and after the ERM surgery in the 28 eyes.

The preoperative logMAR BCVA was $0.23 \pm 0.13$ (range, 0.097-0.52). The logMAR BCVA was reduced to $0.40 \pm 0.24(P<0.001$, paired $t$-test $)$ at 1 week postoperatively and then improved to $0.12 \pm 0.15$ at 1 month $(P=0.003)$ and $0.044 \pm 0.13$ at 3 months $(P<0.001)$ after the surgery.

The pre-and postoperative NAT score, vertical score of M-CHARTS (MV) and horizontal score of M-CHARTS (MH) were shown in Figure 1.

The vertical and horizontal distances of the retinal crossings in the IR images were increased postoperatively. The preoperative vertical distance was $3116.69 \pm 962.48 \mu \mathrm{m}$ (range, 1509-5000) which increased by $7.12 \pm 9.58 \%$ from the baseline at 1 month $(P<0.001$, paired $t$-test) and $8.69 \pm 10.72 \%$ from the baseline at 3 months $(P<0.001)$. The preoperative horizontal distance was $3032.28 \pm 1137.50 \mu \mathrm{m}$ (range, 1346-5810) which increased by $5.60 \pm 9.18 \%$ from the baseline at 1 month $(P=0.007)$ and $8.02 \pm 10.86 \%$ from the baseline at 3 months $(P=0.001)$.

The pre-and postoperative NAT scores and BCVA were not significantly correlated at any time.

The correlations between the ratios of the retinal displacements and the NAT scores are shown in Table 1. The preoperative NAT was significantly correlated with the ratio of the vertical retinal displacement at 1 month $(r=0.43 ; P=0.024)$ and at 3 months $(r=0.49 ; P=0.010)$. The postoperative NAT at 3 months was significantly correlated with the vertical displacement at 3 months $(r=0.54 ; P=0.005)$.

The correlations between the NAT scores and M-CHARTS scores are shown in Table 2. The preoperative NAT was significantly correlated with the preoperative $\mathrm{MH}(r=0.61 ; P=0.001)$. The postoperative NAT at 3 months was significantly correlated with the postoperative $\mathrm{MH}$ and $\mathrm{MV}$ at 3 months (MH: $r=0.49$; $P=0.012, \mathrm{MV}: r=0.40 ; P=0.040$ ).

A representative case is shown in Figure 2.

\section{Discussion}

Our results showed that the pre- and postoperative aniseikonia scores were significantly correlated with the pre- and postoperative metamorphopsia score and with the ratio of the tangential retinal displacements after the surgical removal of an ERM. Although there was a similar study that report retinal displacement in ERM with the measurement of retinal blood vessels, ${ }^{9}$ this was the first study that examined an association between metamorphopsia and aniseikonia in ERM. Although the degree of aniseikonia did not improve at any postoperative times, the visual acuity and the degree of
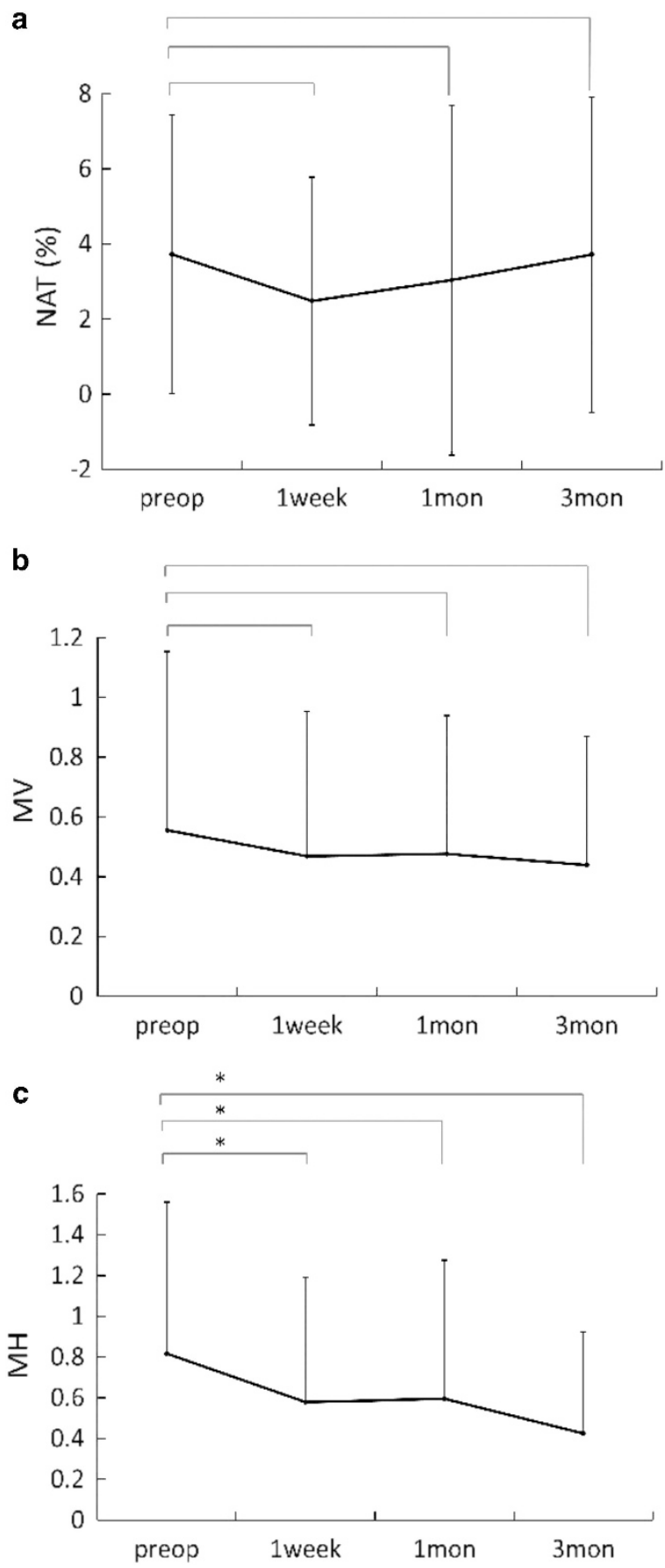

Figure 1 (a) Graph shows the changes of the NAT after epiretinal membrane surgery. The preoperative NAT score was $3.72 \pm 3.71 \%$ (range, 1-15). NAT did not significantly improve at any postoperative times $(P=0.15$ at 1 week, $P=0.10$ at 1 month, $P=0.84$ at 3 months). (b) Graph shows the changes of the MV after epiretinal membrane surgery. The preoperative MV was $0.56 \pm 0.60$ degree (range, 0-2.0). MV did not improve significantly at any postoperative times $(P=0.52$ at 1 week, $P=0.36$ at 1 month, $P=0.29$ at 3 months). (c) Graph shows the changes of the $\mathrm{MH}$ after epiretinal membrane surgery. The preoperative $\mathrm{MH}$ was $0.82 \pm 0.74$ (range $0-2.0)$, and it improved to $0.58 \pm 0.61(P=0.009)$ at 1 week, $0.60 \pm 0.68(P=0.019)$ at 1 month, and $0.43 \pm 0.50(P=0.001)$ at 3 months. ${ }^{*}(P<0.05)$ is considered statistically significant. 
Table 1 Correlation of new aniseikonia test score with vertical or horizontal retinal displacement before and after surgery

\begin{tabular}{|c|c|c|c|c|c|c|c|c|c|c|c|c|}
\hline & \multicolumn{2}{|c|}{$\begin{array}{c}\text { Vertical RD\% at } \\
1 \text { Week }\end{array}$} & \multicolumn{2}{|c|}{$\begin{array}{c}\text { Horizontal RD\% at } \\
1 \text { Week }\end{array}$} & \multicolumn{2}{|c|}{$\begin{array}{c}\text { Vertical RD\% at } \\
1 \text { Month }\end{array}$} & \multicolumn{2}{|c|}{$\begin{array}{c}\text { Horizontal } R D \% \text { at } \\
1 \text { Month }\end{array}$} & \multicolumn{2}{|c|}{$\begin{array}{l}\text { Vertical RD\% at } \\
3 \text { Months }\end{array}$} & \multicolumn{2}{|c|}{$\begin{array}{c}\text { Horizontal RD\% at } \\
3 \text { Months }\end{array}$} \\
\hline & $\mathrm{r}$ & $\mathrm{P}$ & $\mathrm{r}$ & $\mathrm{P}$ & $\mathrm{r}$ & $\mathrm{P}$ & $\mathrm{r}$ & $P$ & $\mathrm{r}$ & $\mathrm{P}$ & $\mathrm{r}$ & $\mathrm{P}$ \\
\hline Baseline NAT & 0.307 & 0.118 & 0.157 & 0.422 & 0.428 & $0.024^{\mathrm{a}}$ & 0.148 & 0.434 & 0.488 & $0.010^{\mathrm{a}}$ & 0.250 & 0.185 \\
\hline NAT at 1 Week & 0.203 & 0.302 & 0.103 & 0.598 & & & & & & & & \\
\hline NAT at 1 Month & & & & & 0.242 & 0.227 & 0.044 & 0.826 & & & & \\
\hline NAT at 3 months & & & & & & & & & 0.539 & $0.005^{\mathrm{a}}$ & 0.024 & 0.899 \\
\hline
\end{tabular}

Abbreviations: NAT, new aniseikonia test score; $\mathrm{RD} \%$, ratio of the change of retinal displacement. ${ }^{\mathrm{a}} \mathrm{P}<0.05$ (bold numbers) was considered statistically significant.

Table 2 Correlation of metamorphopsia score with new aniseikonia test score before and after surgery

\begin{tabular}{|c|c|c|c|c|c|c|c|c|}
\hline & \multicolumn{2}{|c|}{ Baseline MV } & \multicolumn{2}{|c|}{$M V$ at 1 week } & \multicolumn{2}{|c|}{$M V$ at 1 month } & \multicolumn{2}{|c|}{$M V$ at 3 months } \\
\hline & $\mathrm{r}$ & $\mathrm{P}$ & $\mathrm{r}$ & $\mathrm{P}$ & $\mathrm{r}$ & $\mathrm{P}$ & $\mathrm{r}$ & $\mathrm{P}$ \\
\hline \multirow{6}{*}{$\begin{array}{l}\text { Baseline NAT } \\
\text { NAT at } 1 \text { Week } \\
\text { NAT at } 1 \text { Month } \\
\text { NAT at } 3 \text { Months }\end{array}$} & 0.313 & 0.097 & 0.414 & $0.028^{\mathrm{a}}$ & 0.430 & $0.031^{\mathrm{a}}$ & 0.252 & 0.190 \\
\hline & & & 0.415 & $0.028^{\mathrm{a}}$ & & & & \\
\hline & & & & & 0.476 & $0.017^{\mathrm{a}}$ & & \\
\hline & & & & & & & 0.396 & $0.040^{\mathrm{a}}$ \\
\hline & \multicolumn{2}{|c|}{ Baseline $M H$} & \multicolumn{2}{|c|}{ MH at 1 Week } & \multicolumn{2}{|c|}{ MH at 1 Month } & \multicolumn{2}{|c|}{ MH at 3 Months } \\
\hline & $\mathrm{r}$ & $\mathrm{P}$ & $\mathrm{r}$ & $\mathrm{P}$ & $\mathrm{r}$ & $\mathrm{P}$ & $\mathrm{r}$ & $\mathrm{P}$ \\
\hline Baseline NAT & 0.614 & $0.001^{\mathrm{a}}$ & 0.441 & $0.020^{\mathrm{a}}$ & 0.446 & $0.026^{\mathrm{a}}$ & 0.365 & 0.058 \\
\hline NAT at 1 Week & & & 0.268 & 0.156 & & & & \\
\hline NAT at 1 Month & & & & & 0.450 & $0.025^{\mathrm{a}}$ & & \\
\hline NAT at 3 Months & & & & & & & 0.486 & $0.012^{\mathrm{a}}$ \\
\hline
\end{tabular}

Abbreviations: MH, horizontal score of M-CHARTS; MV, vertical score of M-CHARTS; NAT, new aniseikonia test score. ${ }^{a} P<0.05$ (bold numbers) was considered statistically significant.

horizontal metamorphopsia improved after the ERM was removed.

Okamoto et $a l^{7,10}$ reported that the preoperative aniseikonia score was significantly correlated with the thicknesses of the retinal ganglion cell (RGC) layer and the inner nuclear layer (INL). In addition, the postoperative aniseikonia scores at 3 months and 6 months were significantly correlated with the INL thickness. They concluded that the INL thickness was a good indicator of the degree of aniseikonia both before and after surgery and could be used to predict the aniseikonia score in patients that had undergone ERM surgery. These findings can be explained by our results. A thickened INL can result from a tangential shrinkage of the inner retina.

To explain the reason why shrinkage of the inner retina causes aniseikonia and metamorphopsia, we used the explanation that the retinal glial cells act as optical fibers that transmit incoming light through the retinal surface to the photoreceptors. ${ }^{5,11}$ If the inner retina is displaced more than photoreceptor layers, then photons that enter Müller cells reach photoreceptors located away from the original cells. These irregular activations of the photoreceptors may contribute to the generation of the metamorphopsia.

Ooto et $a l^{12}$ showed the results of high-resolution imaging of the photoreceptor layer in eyes with an ERM by using adaptive optics scanning laser ophthalmoloscopy. They found abnormal cone mosaic patterns and microfolds in the photoreceptor layer. The results showed that shrinkage of inner retina caused dislocations of the photoreceptors. Because the aniseikonia did not improve postoperatively in our patients, the compressed photoreceptors are less likely to become normal after surgery which would then generate the perception of macropsia.

There are limitations in this study. This was a retrospective study of a limited number of participants. In addition, the movements of the retinal vessels and retinal thickness were measured manually. We examined the NAT using only the vertically placed semicircle. Previous studies reported that many of patients with ERM had substantial differences in vertical and horizontal aniseikonia, ${ }^{2}$ and that vertical aniseikonia score was 


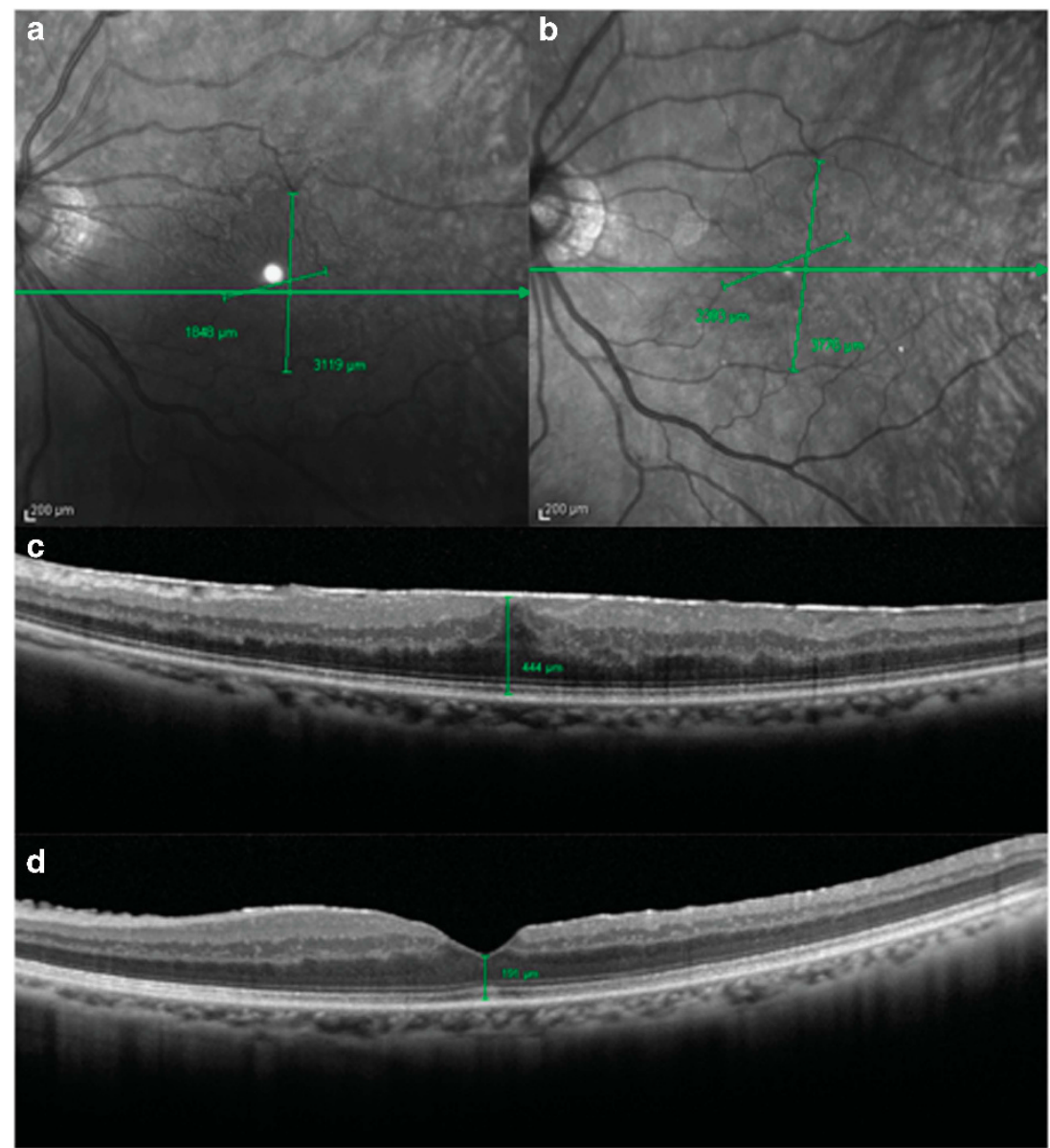

Figure 2 A 64-year-old man with an idiopathic ERM. His preoperative BCVA was 20/32, and the preoperative New Aniseikonia Test score was $5 \%$. The preoperative vertical and horizontal scores of M-CHARTS were 0.7 and 0.5 , respectively. Near infrared image of the eye with an ERM. The distances between 2 intersections of retinal vessels were measured in the near infrared image such as this. (a) Preoperative vertical distance was $3119 \mu \mathrm{m}$ and preoperative horizontal distance was $1848 \mu \mathrm{m}$. (b) The distances between the two intersections of retinal vessels were measured 3 months postoperatively. Postoperative visual acuity was 20/20. Postoperative New Aniseikonia Test score was $4 \%$. Postoperative vertical and horizontal scores of M-CHARTS were 0.9 and 0.2 , respectively. Postoperative vertical distance was $3776 \mu \mathrm{m}$ and postoperative horizontal distance was $2393 \mu \mathrm{m}$. (c) Preoperative optical coherence tomographic image. The preoperative central foveal thickness was $444 \mu \mathrm{m}$. (d) Optical coherence tomographic image 3 months after vitrectomy. Postoperative central foveal thickness was $191 \mu \mathrm{m}$.

larger than horizontal aniseikonia score, although it was not significant. ${ }^{8}$

In conclusion, the results showed that the aniseikonia was less likely to recover after ERM surgery, but the degree of aniseikonia was significantly correlated with the metamorphopsia score, and the distance of tangential retinal displacement in eyes with ERM. Because the purpose of ERM surgery is to remove tractions on the retinal surface, measurement of metamorphopsia is useful for the evaluation of corrected positions of Müller cells after the surgery. Considering that aniseikonia is less likely to improve, we recommend that patients have ERM surgery while the photoreceptors are not severely displaced.

\section{Summary}

What was known before

- Epiretinal membrane causes metamorphopsia and/or aniseikonia.

- The severity of metamorphopsia is related to the tangential retinal contraction.

What this study adds

- Metamorphopsia is significantly correlated with aniseikonia.

- The severity of aniseikonia is also related to the tangential retinal contraction.

- Metamorphopsia is more likely to improve than aniseikonia after epiretinal membrane surgery. 


\section{Conflict of interest}

The authors declare no conflict of interest.

\section{Acknowledgements}

YI is funded by the grant from Koreisha-GanshikkanKenkyu-Zaidan in Japan.

\section{References}

1 Benegas NM, Egbert J, Engel WK, Kushner BJ. Diplopia secondary to aniseikonia associated with macular disease. Arch Ophthalmol 1999; 117: 896-899.

2 Ugarte M, Williamson TH. Aniseikonia associated with epiretinal membranes. Br J Ophthalmol 2005; 89: 1576-1580.

3 Rutstein RP. Retinally induced aniseikonia:a case series. Optom Vis Sci 2012; 89: 50-55.

4 de Wit GC, Muraki CS. Field-dependent aniseikonia associated with an epiretinal membrane a case study. Ophthalmology 2006; 113: 58-62.

5 Ichikawa Y, Imamura Y, Ishida M. Metamorphopsia and tangential retinal displacement after epiretinal membrane surgery. Retina 2017; 37: 673-679.

6 Katsumi O, Miyanaga Y, Hirose T, Okuno H, Asaoka I. Binocular function in unilateral aphakia. Correlation with aniseikonia and stereoacuity. Ophthalmology 1988; 95: 1088-1093.

7 Okamoto F, Sugiura Y, Okamoto Y, Hiraoka T, Oshika T. Time course of changes in aniseikonia and foveal microstructure after vitrectomy for epiretinal membrane. Ophthalmology 2014; 121: 2255-2260.

8 Chung H, Son G, Hwang DJ, Lee K, Park Y, Sohn J. Relationship between vertical and horizontal aniseikonia scores and vertical and horizontal OCT images in idiopathic epiretinal membrane. Invest Ophthalmol Vis Sci 2015; 56: 6542-6548.

9 Rodrigues IA, Lee EJ, Williamson TH. Measurement of retinal displacement and metamorphopsia after epiretinal membrane or macular hole surgery. Retina 2016; 36: 695-702.

10 Okamoto F, Sugiura Y, Okamoto Y, Hiraoka T, Oshika T. Inner nuclear layer thickness as a prognostic factor for metamorphopsia after epiretinal membrane surgery. Retina 2015; 35: 2107-2114

11 Labin AM, Safuri SK, Ribak EN, Perlman I. Müller cells separates between wavelengths to improve day vison with minimal effect upon night vision. Nature communications 2014; 5: 4319.

12 Ooto S, Hangai M, Takayama K, Sakamoto A, Tsujikawa A, Oshima $\mathrm{S}$ et al. High-resolution imaging of the photoreceptor layer in epiretinal membrane using adaptive optics scanning laser ophthalmoscopy. Ophthalmology 2011; 118: 873-881. 